\title{
OBTENÇÃO DE ADI POR TRATAMENTO DE AUSTÊMPERA UTILIZANDO BANHO DE ZAMAC 5 *
}

\author{
Leonardo Pereira ${ }^{1}$ \\ Luiz Francisco Seibel Júnior ${ }^{2}$ \\ Willian Martins Pasini ${ }^{3}$ \\ Regis Fabiano do Amaral $^{4}$ \\ Vinicius Karlinski de Barcellos ${ }^{5}$
}

\section{Resumo}

Este trabalho reporta a viabilidade de realizar o tratamento térmico de austêmpera, para obtenção de um Ferro Dúctil Austemperado (ADI), utilizando uma liga de Zn-Al$\mathrm{Mg}-\mathrm{Cu}$, como meio de resfriamento e permanência em patamar, durante o processo de tratamento térmico. Por meio da obtenção da curva de resfriamento da liga Zamac 5, constatou-se a viabilidade de realização da austêmpera em temperaturas superiores a $380^{\circ} \mathrm{C}$. A partir de corpos de prova de ferro fundido nodular de matriz predominantemente perlítica, instrumentados e austenitizados a $870^{\circ} \mathrm{C}$ e $900^{\circ} \mathrm{C}$, avaliou-se a taxa de resfriamento promovida pela liga Zamac 5, até a temperatura de $400^{\circ} \mathrm{C}$. O tratamento mostrou-se eficiente, utilizando a liga como meio de austêmpera, sendo registradas taxas de resfriamento superiores a $6^{\circ} \mathrm{C} / \mathrm{s}$. A análise morfológica constatou a formação de ausferrita, sem a presença de perlita, caracterizando assim a formação do ADI.

Palavras-chave: Austêmpera; Zamac; ADI; Ausferrita

\section{OBTAINMENT OF ADI BY AUSTEMPERING TREATMENT USING ZAMAK 5 BATH}

\section{Abstract}

This work reports the feasibility of conducting austempering heat treatment to obtain an Austempered Ductile Iron (ADI), using a $\mathrm{Zn}-\mathrm{Al}-\mathrm{Mg}-\mathrm{Cu}$ alloy, as means of cooling and sustaining the thermal arrest during the heat treatment process. By obtaining the alloy cooling curve, with a composition corresponding to Zamak 5, it was verified the feasibility of austempering at temperatures above $380^{\circ} \mathrm{C}$. From samples of nodular cast iron with predominantly perlite matrix, instrumented and austenitized at $870{ }^{\circ} \mathrm{C}$ and $900^{\circ} \mathrm{C}$, the cooling rate promoted by the Zamak 5 alloy was evaluated, until the temperature of $400{ }^{\circ} \mathrm{C}$. The treatment was efficient, using the alloy as austempering medium, with cooling rates higher than $6{ }^{\circ} \mathrm{C} / \mathrm{s}$. The morphological analysis verified the formation of ausferrite, without the presence of perlite, characterizing the formation of ADI.

Keywords: Austempering; Zamak; ADI; Ausferrite

1 Graduando em Engenharia Metalúrgica, Laboratório de Fundição - LAFUN, Universidade Federal do Rio Grande do Sul, Porto Alegre, Rio Grande do Sul, Brasil.

2 Engenheiro Mecânico, Mestrando em Engenharia, Laboratório de Fundição - LAFUN, Universidade Federal do Rio Grande do Sul, Porto Alegre, Rio Grande do Sul, Brasil.

3 Engenheiro Metalúrgico, Mestrando em Engenharia, Laboratório de Fundição - LAFUN, Universidade Federal do Rio Grande do Sul, Porto Alegre, Rio Grande do Sul, Brasil.

4 Engenheiro Metalúrgico, Mestre em Engenharia, Técnico Científico, Laboratório de Fundição LAFUN, Universidade Federal do Rio Grande do Sul, Porto Alegre, Rio Grande do Sul, Brasil.

5 Engenheiro Metalúrgico, Doutor em Engenharia, Professor, Laboratório de Fundição - LAFUN, Universidade Federal do Rio Grande do Sul, Porto Alegre, Rio Grande do Sul, Brasil. 


\section{INTRODUÇÃO}

A fabricação de peças por processo de fundição surgiu a mais de 4000 anos a.C., as técnicas empregadas tiveram enorme evolução, e ainda são objeto de investigação e inovação. Por ser um processo de fabricação amplo e versátil, encontram-se itens fabricados por fundição desde objetos com finalidades artísticas a itens de alta responsabilidade com aplicações médicas e aeroespaciais.

Conforme Keough e Kathy [1] o tratamento de austêmpera foi desenvolvido na década de 1930. O ferro fundido nodular ou dúctil, foi obtido na década seguinte. Em 1970 se inicia a fabricação de ADI, o qual registrou aumento significativo da sua produção nos anos posteriores. Atualmente uma variedade enorme de peças são fabricadas em ADI. A combinação, das vantagens do processo de fabricação por fundição e das propriedades do material, possibilitaram o desenvolvimento de componentes que antes eram obtidos somente por forjamento, usinagem ou soldagem.

Nas últimas décadas o desenvolvimento e aperfeiçoamento do ferro fundido nodular austemperado (ADI), fez surgir novas possibilidades para engenheiros projetistas, isso decorre das combinações singulares deste material. O ADI combina propriedades como a resistência mecânica e tenacidade de maneira única [2], ambas associadas também, à elevada resistência ao desgaste [3]. É amplamente conhecido no meio metalúrgico e industrial através da sigla ADI, utilizada para denominação de "Austempered Ductile Iron".

A microestrutura característica do ADI é a ausferrita. Essa estrutura singular é resultado do ferro fundido nodular, submetido ao tratamento isotérmico de austêmpera.

A combinação de densidade do ferro nodular e propriedades mecânicas do ADI, fazem do mesmo um substituto viável para as ligas de alumínio, por exemplo, onde a robustez do ADI, garante performance de propriedades permitindo redução de peso do componente. Engrenagens, virabrequins, eixo de came, cubos de roda são alguns exemplos de componentes que podem ser fabricados em ADI. Existem muitos outros que encontram sua aplicação na indústria automobilística, de equipamentos agrícolas, de maquinas e ferramentas e do setor de mineração [3].

Para a obtenção de um componente em ADI é necessário um controle rígido de todas as suas variáveis de fabricação. Essas variáveis dizem respeito à composição química da liga, geometria da peça, o processo de fundição e os parâmetros do ciclo de tratamento térmico utilizado, que são: temperatura e tempo de austenitização e de austêmpera, assim como a taxa de resfriamento [4].

Austêmpera é realizada usualmente na faixa de temperatura compreendida entre $260^{\circ} \mathrm{C}$ e $420^{\circ} \mathrm{C}$. O tratamento habitualmente é realizado em banho de sais fundidos, que necessitam de uma série de cuidados. As particularidades do banho de sais levam ao encarecimento do tratamento, devido a questões de: Licenças ambientais e de fiscalização governamental, logística do processo, de recuperação e destinação de resíduos pós-tratamento.

Existem alguns metais e suas ligas, que são líquidos na faixa de temperatura usual de austêmpera, podendo em tese serem utilizados como meio de tratamento isotérmico. O Zamac 5, é uma liga essencialmente de Zinco, Alumínio, Cobre e Magnésio, com boa disponibilidade no mercado visto que é utilizada para fabricação de diversos itens. A possibilidade de realização de austêmpera em uma liga eutética de zinco e alumínio já foi reportada na literatura [4], no qual a austêmpera realizouse na faixa de $400^{\circ} \mathrm{C}$. 
Nenhum parâmetro do processo de fabricação do ADI deve ser analisado individualmente, mas é esperado que baixas temperaturas de austêmpera, menor que $350^{\circ} \mathrm{C}$, contribui para obtenção de ADI de classes maiores como a ASTM III, IV e V [5], onde os ganhos de resistência mecânica e dureza são mais significativos que os ganhos em ductilidade e tenacidade. Por outro lado, quando utiliza-se altas temperaturas de austêmpera, maior que $350^{\circ} \mathrm{C}$, se obtém ADI em classes ASTM I e II, cujos incrementos relacionados à tenacidade e ductilidade são mais expressivos do que os relacionados à resistência mecânica [6].

A redução da temperatura de austêmpera diminui as dimensões das agulhas de ferrita [7], onde esta condição gera aumento da resistência mecânica por redução do espaço livre associado ao caminho para movimentação de discordâncias [8]. Ainda como fator adicional, a redução da temperatura de austêmpera, propicia a diminuição da quantidade de austenita inclusa na ausferrita, aumentando a quantidade de ferrita [7].

Em ligas de $\mathrm{Zn}-\mathrm{Al}-\mathrm{Cu}-\mathrm{Mg}$, o magnésio atua diminuindo a temperatura de líquidos, já o cobre reduz a oxidação [9-10]. Neste estudo verificou-se a possibilidade da utilização do Zamac 5, como uma alternativa ao banho de sais, na realização da austêmpera para obtenção de ADI.

\section{MATERIAIS E MÉTODOS}

O Zamac 5 foi utilizado em forma de lingotes comerciais, teve sua composição química analisada em espectrômetro de emissão óptica, cujo resultado se encontra na Tabela 1.

Tabela 1 - Composição química do ZAMAC 5 utilizado (\% em peso)

\begin{tabular}{ccccccc}
\hline $\mathbf{A l}$ & $\mathbf{C u}$ & $\mathbf{M g}$ & $\mathbf{F e}$ & $\mathbf{S i}$ & $\mathbf{Z n}$ & Outros \\
\hline 4,24 & 1,9 & 0,12 & 0,3 & 0,02 & 93,4 & 0,02 \\
\hline
\end{tabular}

O lingote foi fracionado de modo a permitir a introdução em um cadinho de carbeto de silício, com capacidade de $325 \mathrm{ml}$.

Foram confeccionados 02 (dois) corpos de prova (CPs) de ferro fundido nodular de matriz perlítica, proveniente de peças brutas de fusão. A liga utilizada nestas peças é própria para aplicação do tratamento térmico de austêmpera, com objetivo de obter ADI. A composição química da liga é descrita na Tabela 2.

Tabela 2 - Composição química do Ferro Fundido Nodular (\% em peso)

\begin{tabular}{cccccc}
\hline $\mathbf{C}$ & $\mathbf{S i}$ & $\mathbf{M n}$ & $\mathbf{M g}$ & $\mathbf{C u}$ & $\mathbf{N i}$ \\
\hline 3,56 & 2,30 & 0,32 & 0,034 & 0,66 & 0,53 \\
\hline $\mathbf{M o}$ & $\mathbf{S n}$ & $\mathbf{S}$ & $\mathbf{P}$ & $\mathbf{C r}$ & $\mathbf{F e}$ \\
\hline 0,18 & 0,02 & 0,007 & 0,021 & 0,03 & Bal. \\
\hline
\end{tabular}

Os CPs foram cortados na forma retangular com os lados medindo $14 \mathrm{~mm}$ e $\mathrm{o}$ comprimento de $44 \mathrm{~mm}$, como mostra a Figura 1. Os corpos de prova em ferro nodular foram preparados com a realização de furação, condição esta que permitiu introduzir os termopares até o centro da peça, local onde o resfriamento é o mais lento. Após a furação, o CP1 pesou 59,4 g e o CP2 70,6 g. 


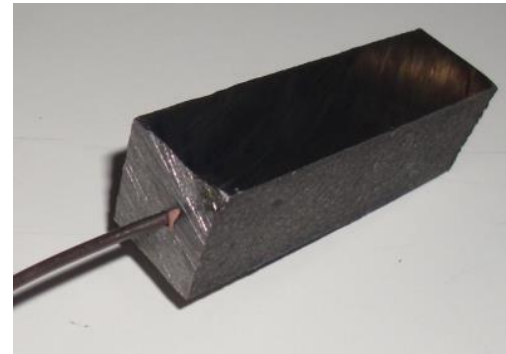

Figura 1 - Corpo de Prova instrumentado.

Em uma etapa inicial, realizou-se a análise térmica da liga Zamac 5 para obtenção da curva de solidificação, a fim de determinar a temperatura liquidus da liga. 0 metal líquido foi vazado em um copo de análise térmica instrumentado com termopar tipo K, conforme mostrado na Figura 2. A aquisição dos dados foi realizado utilizando o software myPcLab-NOVUS, com taxa de aquisição de $4 \mathrm{~Hz}$.

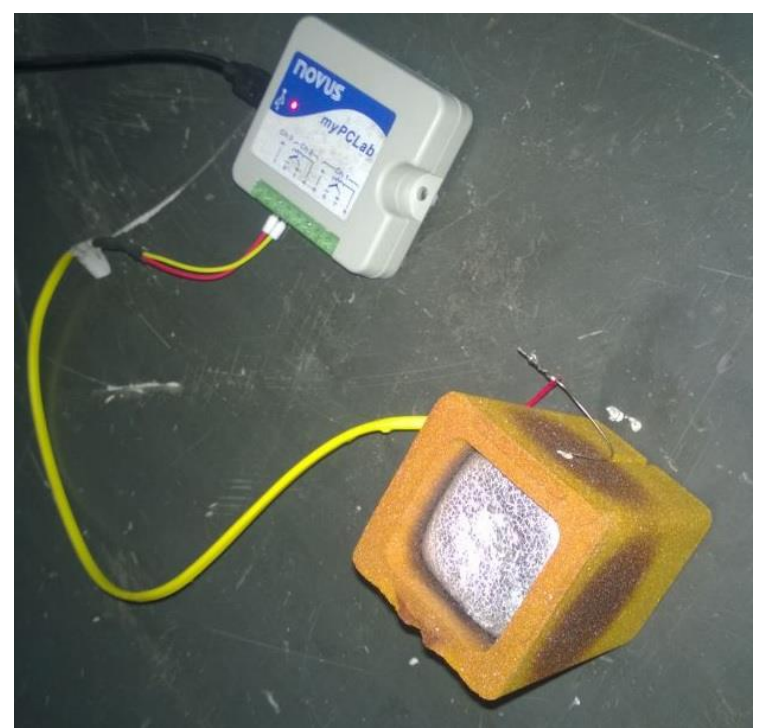

Figura 2 - Copo de análise térmica com Zamac 5 após solidificação.

O tratamento térmico de austenitização foi realizado em forno elétrico resistivo do tipo mufla, aquecido previamente.

O tratamento de austêmpera foi realizado utilizando-se 1,6Kg de Zamac 5. A liga foi fundida em forno resistivo do tipo poço. A temperatura do banho foi mantida em $380^{\circ} \mathrm{C}$.

O tratamento de austenitização foi realizado em duas etapas, que consistem na manutenção do $\mathrm{CP}$ em dois patamares isotérmicos de temperatura, com vistas a minimizar o crescimento de grão. O CP1 foi mantido por 30 minutos no patamar de $850^{\circ} \mathrm{C}$ e posteriormente por mais 30 minutos à $880^{\circ} \mathrm{C}$. $\mathrm{O} \mathrm{CP} 2$ foi mantido 25 minutos no patamar de $850^{\circ} \mathrm{C}$ e posteriormente por 25 minutos em $900^{\circ} \mathrm{C}$.

Após a etapa de austenitização ser completada, os CPs foram conduzidos imediatamente para o banho metálico fundido de Zamac 5 para realização do tratamento de austêmpera. O tempo de austêmpera foi de aproximadamente 50 min. para o CP1 e de 45 min. para o CP2.

\section{RESULTADOS E DISCUSSÃO}

\subsection{Curva de solidificação do ZAMAC 5}


A Figura 3 mostra a curva de resfriamento da liga Zamac 5 indicando a temperatura liquidus e solidus, de $384^{\circ} \mathrm{C}$ e $336^{\circ} \mathrm{C}$, respectivamente.

$\mathrm{Em} 380^{\circ} \mathrm{C}$, temperatura objetivada para o tratamento de austêmpera, ainda se observou uma boa molhabilidade, mesmo com a presença de uma pequena fração de sólido no banho fundido.

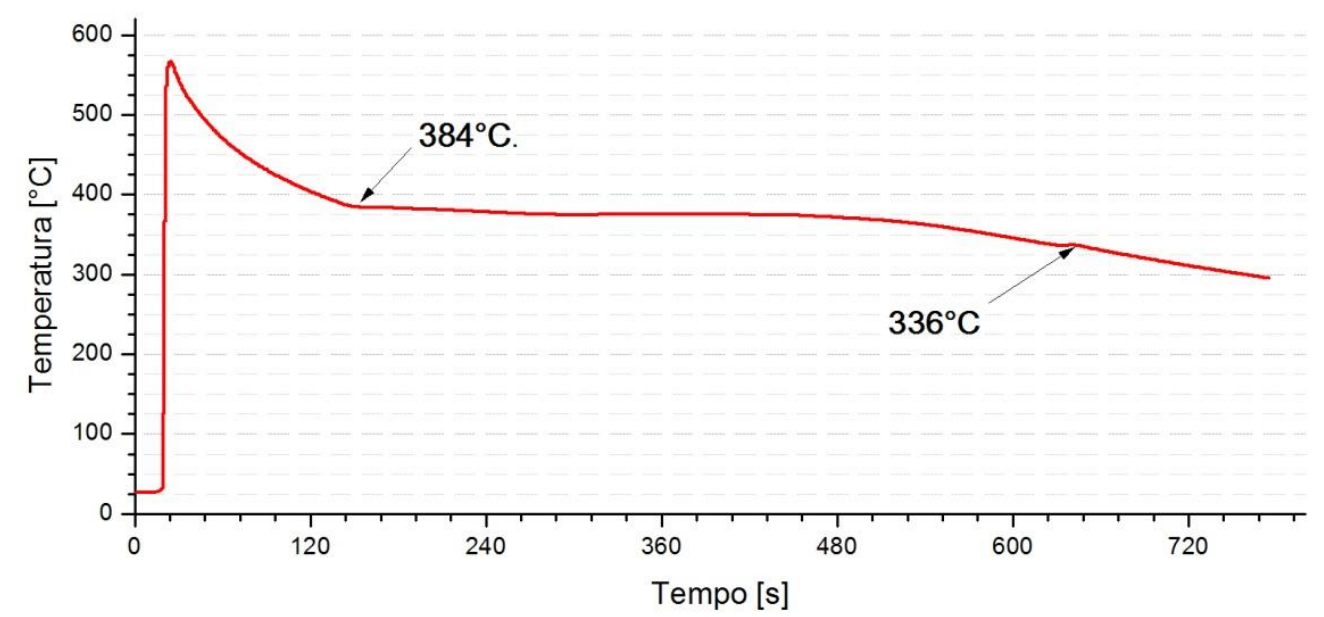

Figura 3 - Curva de resfriamento do ZAMAC 5.

A temperatura de austêmpera pode ser considerada como a variável de grande criticidade para obtenção de valores adequados relacionados à resistência mecânica [11-12].

\subsection{Taxa de resfriamento do CPs}

Deve-se pensar que ao introduzir o CP austenizado no banho é esperado um aumento significativo da temperatura deste. Entretanto isso não foi observado, como observa-se nas Figuras 4 e 5, devido a presença de fração sólida na temperatura de $380^{\circ} \mathrm{C}$. O calor liberado pelo $\mathrm{CP}$ ocasionou pequena elevação da temperatura, e consequentemente a fusão da fração sólida.

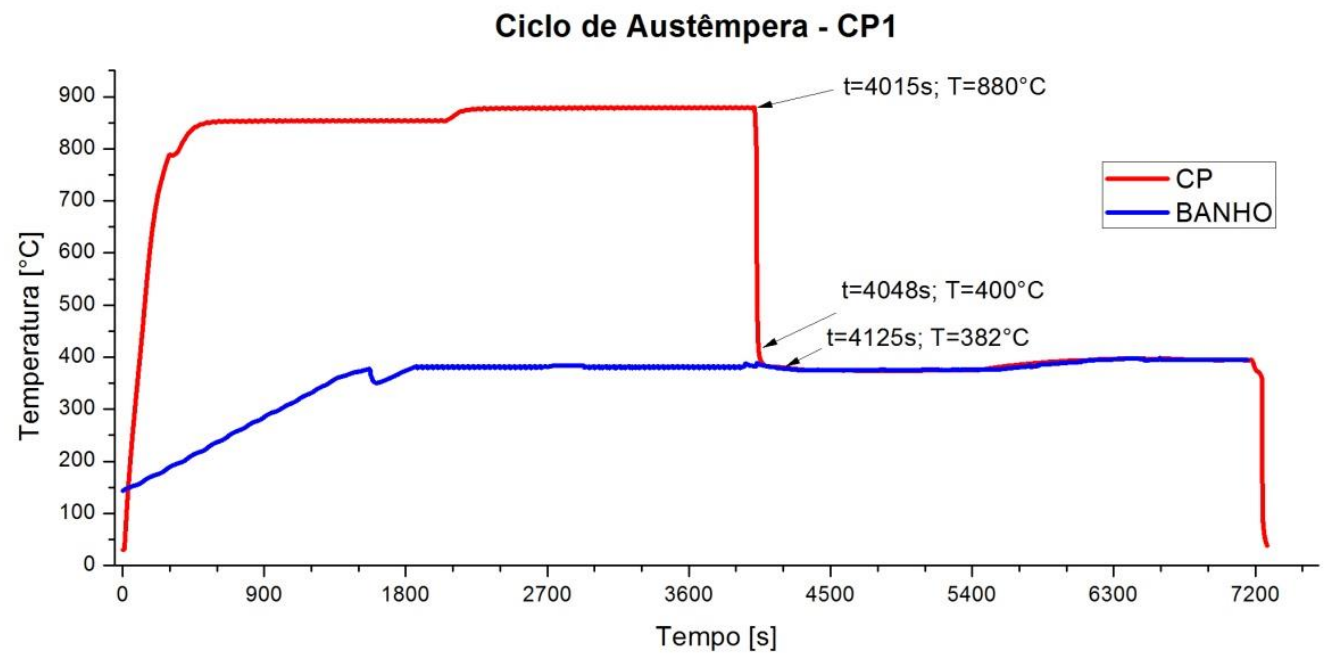

Figura 4 - Gráfico do Ciclo de austêmpera do CP1. 
Para o CP1 o resfriamento entre $880^{\circ} \mathrm{C}$ e $400^{\circ} \mathrm{C}$ ocorreu a uma taxa média de $14,5^{\circ} \mathrm{C} / \mathrm{s}$. Após o fim do tratamento isotérmico, o CP1 foi resfriado em água.

Variações consideráveis na temperatura do banho ocorreram antes da realização da austêmpera, como observado na Figura 4, decorrente do ajuste da temperatura do forno nesse período. Após o ajuste, foi possível manter a temperatura estabilizada.

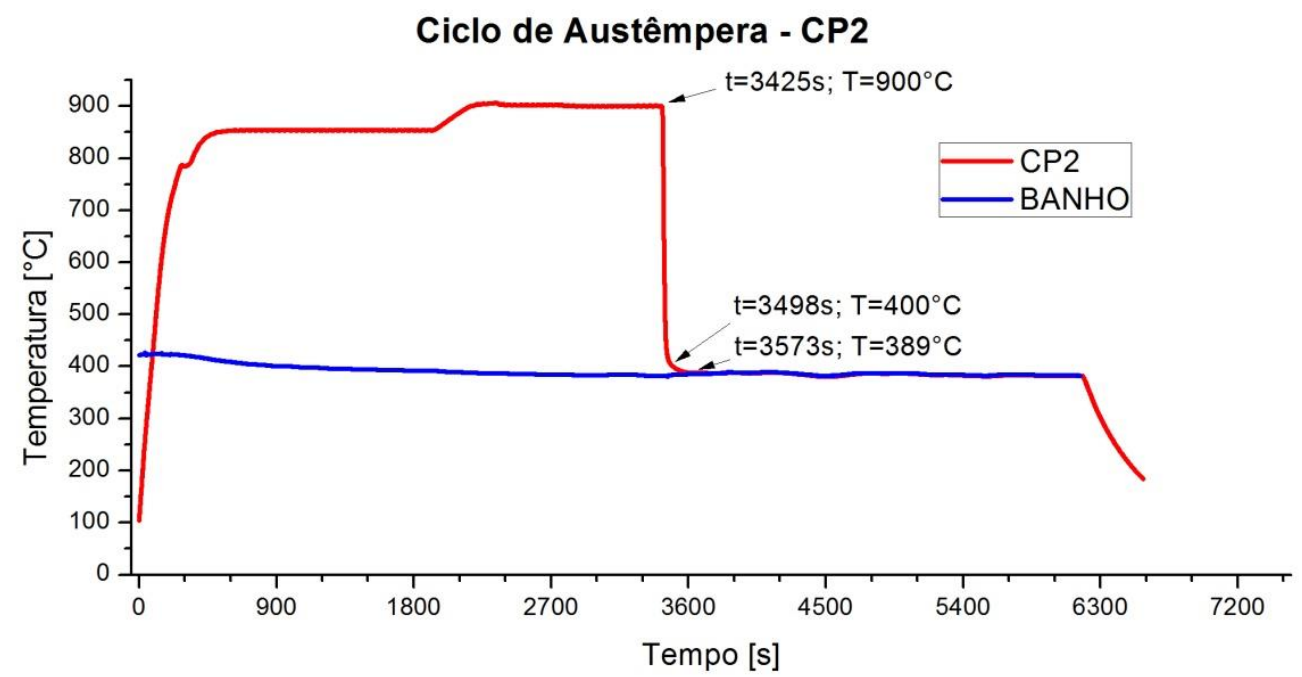

Figura 5 - Gráfico do Ciclo de Austêmpera do CP2.

Para o CP2 o resfriamento entre $900^{\circ} \mathrm{C}$ e $400^{\circ} \mathrm{C}$ ocorreu à taxa média de $6,85^{\circ} \mathrm{C} / \mathrm{s}$. Após o tratamento isotérmico, o CP2 foi resfriado ao ar.

As menores taxas de resfriamento observadas no CP2 em relação ao CP1 são resultantes dos seguintes fatores:

1. CP2 estava a uma temperatura superior;

2. CP1 possuía menos massa;

3. O banho se encontrava em uma temperatura ligeiramente superior.

Não houve premissa em uniformizar a temperatura de austenitização dos corpos de prova, porque o objetivo era avaliar a eficiência do banho, e não estabelecer comparativos entre os diferentes parâmetros de austenitização.

\subsection{Microestrutura dos CPs}

Após a austenitização, ocorre a seguinte sequência de eventos, que leva a formação da ausferrita [4]:

1. Resfriamento rápido até a temperatura de austêmpera, de modo a evitar que ocorra a formação da estrutura perlítica;

2. A partir do grão austenítico metaestável, que contem entre 1,2 e 1,6\% de carbono, ocorre à nucleação da ferrita acicular;

3. Inicia-se o crescimento de grão ferrítico, a difusão eleva o teor de carbono na austenita para a faixa de 1,8 a 2,2\%, conferindo a austenita estabilidade mecânica e térmica;

4. Interrompe-se o tratamento isotérmico de austêmpera, de modo a evitar a degradação da austenita de alto carbono, que caso ocorresse, resultaria em ferrita e carbonetos.

Observando-se as Figuras 6 e 7, é averiguável que em ambos os ciclos de tratamento, foi obtida a microestrutura caracteriza do ADI. A solução de Nital a $2 \%$ 
utilizada para revelar a morfologia da estrutura, ataca preferencialmente a ferrita, de modo que as tonalidades brancas da austenita de alto carbono são facilmente identificadas.

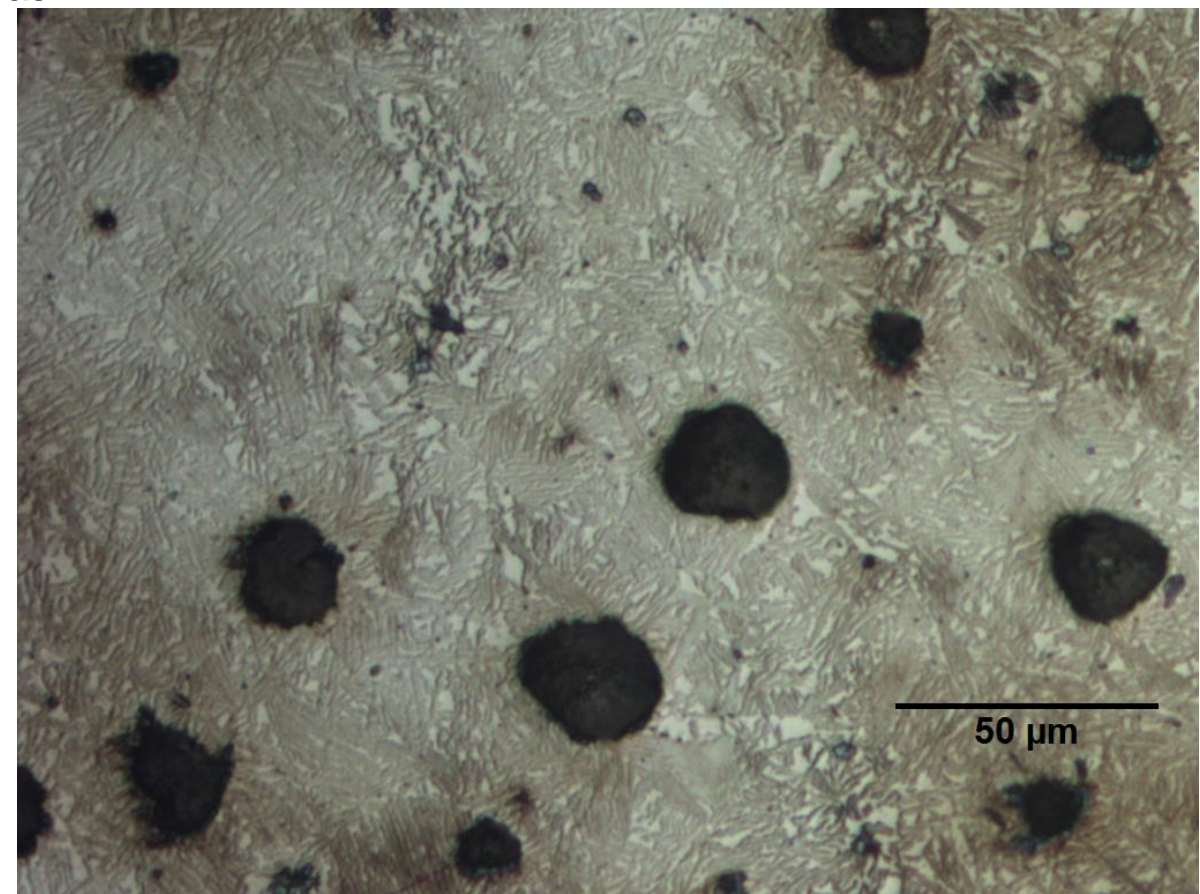

Figura 6 - Micrografias do CP1.

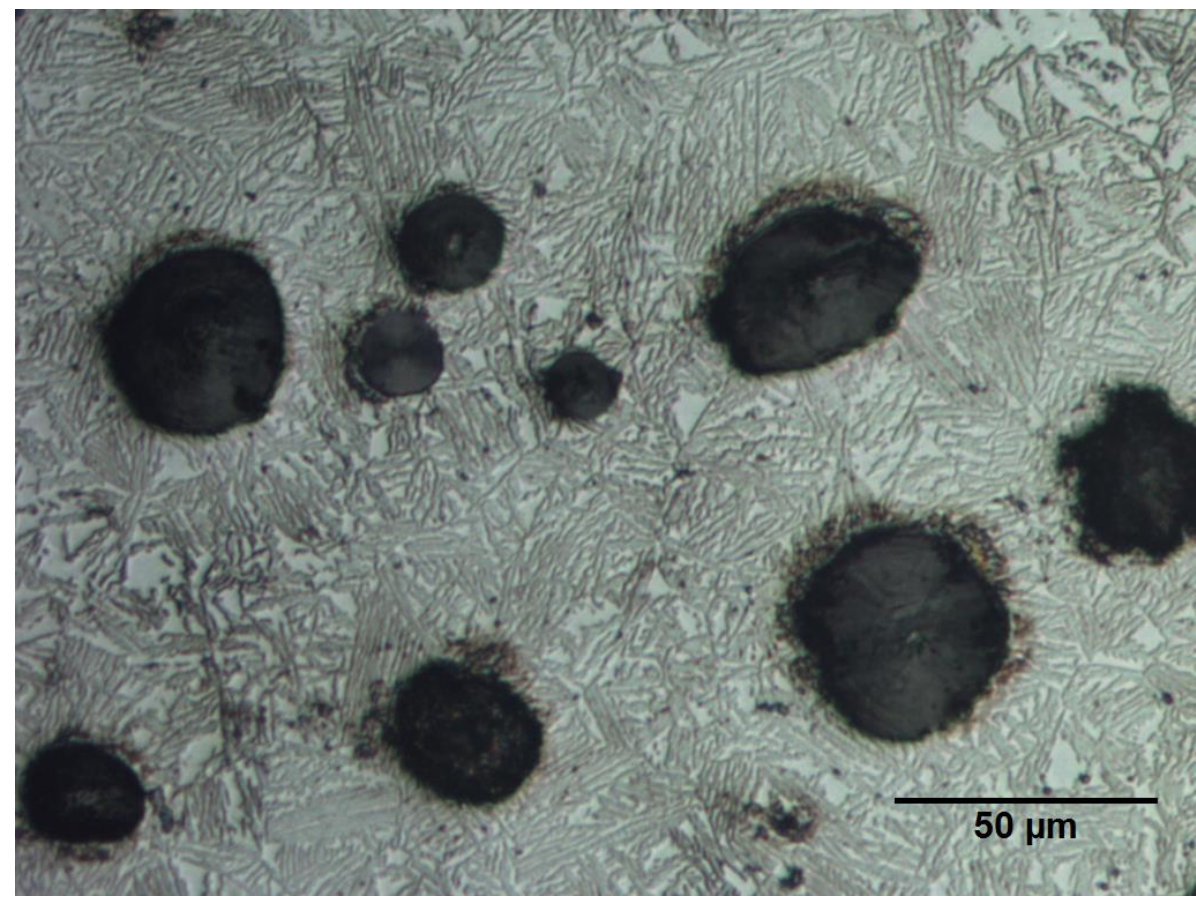

Figura 7 - Micrografias do CP2.

\section{CONCLUSÃO}

O ZAMAC se mostrou um meio satisfatório para realizar o resfriamento do corpo de prova aquecido na temperatura de austenitização. Não foi observado à formação de perlita, fato que indicaria uma taxa de resfriamento lenta. O banho de 
Zamac 5 tem potencial adequado para manutenção da temperatura durante todo o tratamento de austêmpera.

Existe uma limitação quanto à faixa de temperatura, em que pode ser realizado o tratamento de austêmpera com o ZAMAC. Enquanto banhos de sais comerciais podem ser utilizados em toda a faixa de temperaturas usuais na austêmpera, o Zamac fica limitado a temperaturas superiores a $375^{\circ} \mathrm{C}$.

\section{REFERÊNCIAS}

1 Keough, John R., and Kathy L. Hayrynen. Automotive Applications of Austempered Ductile Iron (ADI): A Critical Review. No. 2000-01-0764. SAE Technical Paper, 2000.

2 Kovacs, B. V. Development of austempered ductile iron (ADI) for automobile crankshafts. Journal of Heat Treating 1987;5(1): 55-60.

3 Keough, John R., and Kathy L. Hayrynen. Automotive Applications of Austempered Ductile Iron (ADI): A Critical Review. No. 2000-01-0764. SAE Technical Paper, 2000.

4 Souza, Bruno Vaz de. Desenvolvimento de ferro fundido austemperado (ADI) em banho de zinco-alumínio. MS thesis. Pontifícia Universidade Católica do Rio Grande do Sul, 2015.

5 ASTM A897/A897M-06: Standard Specification for Austempered Ductile Iron Castings. American Society for Testing and Materials, ASTM International, USA, 2011.

6 M. Cemal Cakir, Ali Bayrama, Yahya Isik, Baris Salar. The effects of austempering temperature and time onto the machinability of austempered ductile iron." Materials Science and Engineering: 2005;A 407: 147-153.

7 Putatunda, S.K. ; Kesani,S.;Tackett,R.; Lawes ,G - Development of austenite free ADI - Materials Science and Engineering , n 435A, p.112-122, 2006

8 Putatunda, S.K \& Gadicherla, P.K - Influence of austenitizing temperature on fracture toughness of a low manganese ADI - Materials Science and Engineering , $n$ 268A, p.15-31,1999.

9 Krupiński, M., Labisz, K., Tański, T., Krupińska, B., Król, M., \& Polok-Rubiniec, M. Influence of Mg Addition on Crystallisation Kinetics and Structure of the Zn-Al-Cu Alloy. Archives of Metallurgy and Materials, 2016;61(2): 785-790.

10 Rick, Cristiane Fonseca. Estudo da liga à base de Zn-Al-Cu-Mg aplicada na fabricação de jóias folhadas. MS thesis. Pontifícia Universidade Católica do Rio Grande do Sul, 2006.

11 Gundlach,R.B; Janowak,J.F; Bechet,S.;Röhring,K - Transformation Behavior in Austempered Ductile Iron - The Physical Metallurgy of Cast Iron: 1984: p.399-409.

12 Hasse, S. - ADI, um material ideal para produção de peças com paredes espessas. Fundição e Serviços: 1998;Julho: p.60-74. 